\title{
Performance and Analysis of the Altitude for Optical Channel Drones
}

\author{
Abdullah Jameel Mahdi ${ }^{1}$, Wamidh Jalil Mazher ${ }^{2}$, and Osman Nuri Ucan ${ }^{3}$ \\ \{abdullahas396@gmail.com ${ }^{1}$, wamidh.mazher@stu.edu.iq ${ }^{2}$, Osman.Ucan@altinbas.edu.tr ${ }^{3}$ \} \\ Collage of Engineering, Electromech Engineering Department, University of Samarra, Samarra ${ }^{1}$, \\ Thi Qar Technical College, Electrical Technical Department, Southern Technical University, Al-shatraa ${ }^{2}$ \\ Electrical, and Computer Engineering, Altinbas University, Istanbul ${ }^{3}$
}

\begin{abstract}
A drone is a vehicle with no human pilot, staff, or passengers aboard recently used in various civil and military applications. Drones are controlled remotely by a human operator and flown. The technical advantages of free-space optical (FSO) technology motivated the researchers to continue investigating to use it in the communication between drones. Nevertheless, every technology face limiting factors that degrade performance. In this paper, the channel gain $\mathrm{H}$ effect was taken into consideration. The purpose is to compare the performance among different topologies that the system consists of, determine the effect of increase the drone's number on the system impacts, and extract the highest altitude $h$ the system can reach. The system was modelled using MATLAB 2020, and the results showed that as the number of drones increased, the Signal-to-Noise ratio (SNR) increased. The maximum link distance $Z$ between any two parts and the maximum altitude $h$ the system reached was $7800 \mathrm{~m}$.
\end{abstract}

Keywords: Drone, Free space optical, Signal-to-Noise ratio, Channel gain, Altitude, Link distance.

\section{Introduction}

The drone is applying in low-altitude and short-length applications. The drone is one type of unmanned aerial vehicle (UAV), and the latter is part of an unmanned aircraft system (UAS), which needs a ground-based controller [1]. The UAV is classified according to its size and configuration, total take-off weight, operational altitude, ownership, launch system [2]. As control technologies advanced and costs decreased, their utilization expanded quickly and applied in several applications [3] in which some of the drone services available. The FSO technology links the drone and the controller or between the drones themselves. FSO uses the laser to propagate in free space to transmit data wirelessly. Many merits encourage using FSO technology as an alternative to RF technology, such as licensed free, high bit rates, long-range operation, immunity to electromagnetic interference, high security [4], and so on.

On the other hand, many limiting factors existing in both the atmospheric layer and in laser. The atmospheric layer liming factors are fog, rain, pollution, aligned stability in the wind [5], [6]. At the same time, the limiting factors in a laser beam are narrow beamwidth that complicates the alignment between two transceivers, the choice of laser wavelength that is subject to minimal 
losses, etc. As a result of these factors, the receiver signal gets attenuated and limits the valuable distance, resulting in a higher bit error ratio (BER).

In addition to the mentioned limiting factors, the channels connected between drones were classified as a non-stationary link. One significant problem arising from such a link is the pointing error (misalignment) between Tx and Rx. Many studies on systems-based FSO terrestrial links took into account the weather conditions and beamed pointing errors such as [7], [8], and [9]. In contrast, the studies on drone based FSO links are recent and rare, especially in pointing error limiting factors. The study [10] looked at how to improve the UAV system by using relay-assisted to reduce the effects of various atmospheric variables on the FSO signal quality. The research in [11] looked at how the Ground-UAV and UAV-UAV scenarios performed in the presence of air turbulence. As illustrated in [12], the BER goal was met by selecting the optimal beamwidth to reduce transmitted power. The survey [13] focused on the continual movement and changing relative speeds of participating UAV members, making it challenging to establish a line-of-sight (LOS) FSO link in a swarm scenario. The performance of a non-static and tilted link between the stationary station on the ground and the UAV was measured in the study [14]. The study [15] counted the number of drones that may be used to identify and make important decisions for area surveillance (e.g., oil pipeline leak). By describing source-to-relay and relay-to-destination channel models, the study's goal [16] is to calculate the ideal three-dimensional coordinates of the UAV relay and optimal beam pattern to reduce outage probability.

This paper aims to compare the performance among different topologies that the system consists of, determine the effect of increasing the drone number on system performance, and extract the highest altitude $h$ the flying system can reach.

\section{The system configuration}

Drones networking is no longer restricted to point-to-point communication between drones or between drones and remote controllers. Many network configurations can the drone's shape as mentioned in [17] and [18]. In addition, numerous channel kinds, such as Single Input-Single Output (SISO) link, Multiple Input-Single Output (MISO) link, Single Input-Multiple Output (SIMO) link, and Multiple Input-Multiple Output (MIMO) link, can be linked between drones in the network [15], [19] [15], [19]. In this research, the drone swarm's system configuration is the V-shape arrangement, as depicted in Fig 1. Many multi-rotor drones with SISO and MISO channels make up the configuration. The SISO channel is the link between the drones in an arm as well as the channel between the Ground Station (GS) Controller and the Main Drone (DM). The DM is the MISO channel, and it connected the last drones in both arms (D11 and D21). In [15], this V-shape design was used. At the transmitter end, the SISO topology has one transmitting aperture and one receiving aperture. The optical signals can be sent from the MISO's many transmitting antennas. The optical signals from many transmitting antennas are received by just one receiving antenna, implying that different sources are available but only one destination is available [20].

The drones in this study move at a constant speed and alter their altitude $\mathrm{h}$ at the same time. The drones will be on one side, while the GS will be on the other. As shown in Table 1, the system parameters differ for each channel in the system. 


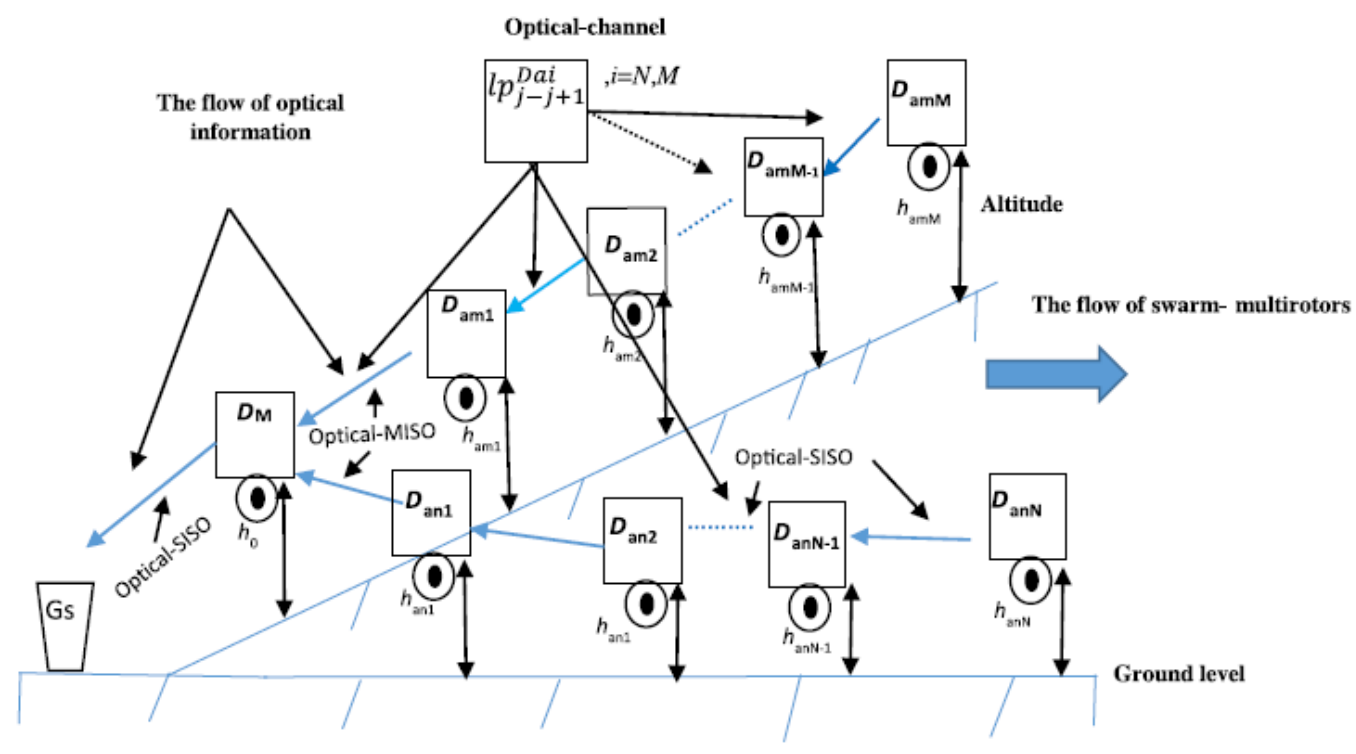

Fig. 1. V-shape swarm FSO-drones perspective illustration [15].

Table 1. The system parameters.

\begin{tabular}{ccc}
\hline Parameter & Symbol & Set to \\
\hline Rate of Transmission & Rate & $1 \mathrm{Gbps}$ \\
OTP & $P_{t}$ & $40 \mathrm{~mW}(16 \mathrm{dBm})$ \\
Responsivity & $R$ & 1 \\
Separated distance & $Z$ & Varying \\
Altitude & $h$ & Varying \\
Wavelength & $\lambda$ & $1550 \mathrm{~nm}$ \\
Receiver Diameter & $D_{R}$ & $1 \mathrm{~cm}$ \\
Pointing Error Angle & $\theta_{r}$ & $1 \times 10^{-5} \mathrm{rad}$ \\
Beam Waist at z=0 & $w_{0}$ & $5 \times 10^{-2} \mathrm{~cm}$ \\
\hline
\end{tabular}

The width $\mathrm{w}_{\mathrm{o}}$ of the transmitted optical beam from the transmitter Tx is measured by the beam waist wo. The $D_{R}$ value of the receiver aperture is $1 \mathrm{~cm}$. It depends on the drone design and weight tolerance; the operating wavelength $\lambda$ is $1550 \mathrm{~nm}$ because it has a smaller attenuation transmission window. The distance $Z$ between two drones or between $\mathrm{D}_{\mathrm{M}}$ and GS was assumed to vary. The altitude of the drones was assumed to vary also.

\section{The Channel mathematical model}

The channel gain $\mathrm{H}$ is made up of three factors: atmospheric attenuation Ha, atmospheric turbulence $\mathrm{H}_{\mathrm{f}}$, and pointing error factor $\mathrm{H}_{\mathrm{p}}$. Each of these factors represents an impairment that faces the FSO system. $\mathrm{H}_{\mathrm{a}}$ and $\mathrm{H}_{\mathrm{f}}$ have modelled the weather condition and the atmosphere's turbulence, and $\mathrm{H}_{\mathrm{P}}$ has modelled the optical beam pointing with channel gain $\mathrm{H}$ : 
$H=\prod_{m=1}^{M} H_{a_{m}} H_{f_{m}} H_{p_{m}}, \quad m=1,2,3, \ldots M$

where $\mathrm{M}$ is the topology's channel count (for SISO, $\mathrm{M}=1$, and MISO=2).

The Beer-Lambert law, represented as [21], has been used to simulate the atmospheric attenuation $\mathrm{H}_{\mathrm{am}}$ :

$\mathrm{H}_{\mathrm{a}_{\mathrm{m}}}=\exp \left(-Z_{m} \sigma\right)$

where $\mathrm{Z}_{\mathrm{m}}$ is the link distance of the mth channel and $\sigma$ is the attenuation coefficient, which is a function of visibility [22]. The channel fading of atmospheric turbulence $\mathrm{H}_{\mathrm{fm}}$ can be expressed as:

$H_{f_{m}}=\frac{2 P_{r_{m}}}{\pi W_{m}}$

Where $\mathrm{P}_{\mathrm{rm}}$ and $\mathrm{W}_{\mathrm{m}}$ are the received optical power and the beam spot radius of the mth channel at the receiver. The pointing error $\mathrm{H}_{\mathrm{pm}}$ of the optical beam between the transmitter and receiver given as:

$H_{p_{m}}=A_{0_{m}} \exp \left(\frac{-2 r_{m}^{2}}{W_{Z e q_{m}}^{2}}\right)$

where optical beam radial displacement is $r_{m}=Z . \theta_{r}^{m}$, pointing error angle is $\theta_{r_{m}}$ of the $m^{\text {th }}$ channel with $\leq 10^{-4}[23], A_{0_{m}}=\left[\operatorname{erf}\left(v_{m}\right)\right]^{2}$ is the fraction of collected power at the radial displacement $r=0$ [24], and beam radius $\mathrm{W}_{\mathrm{Zeq}_{\mathrm{m}}}$ in the $m^{\text {th }}$ channel that can given as [24]:

$W_{Z e q_{m}}=\mathrm{w}_{Z_{m}}^{2} \frac{\sqrt{\pi} \operatorname{erf}\left(v_{m}\right)}{2 \mathrm{v} \exp \left(-v_{m}^{2}\right)}$

where beam radius is $w_{z_{m}}$ at $e^{-2}$ of the axial $Z_{\mathrm{m}}$ distance and beam width $w_{0}$ at $\mathrm{Z}_{\mathrm{m}}=0$ as $w_{z_{m}} \approx$ $w_{0}\left[1+\varepsilon_{m}\left(\lambda Z_{m} / \pi w_{0}^{2}\right)^{2}\right]^{\frac{1}{2}}$, where $\varepsilon_{m}=\left(1+2 \mathrm{w}_{0}^{2} / \rho_{0}^{2}\left(Z_{m}\right)\right)$, and the coherence length $\rho_{0}\left(Z_{m}\right)=\left(0.55 C_{n}^{2}(h) K^{2} Z\right)^{-3 / 5}$, and $v_{m}=(\sqrt{\pi} a) /\left(\sqrt{2} \mathrm{w}_{\mathrm{z}_{\mathrm{m}}}\right)$, where receiver radius is $a$ [25]. The received optical signal [26] is:

$y=x R \sum_{m=1}^{M} H_{m}+v_{n}, \quad m=1,2,3, \ldots . M$

Where $\mathrm{x}$ denotes the transmitted signal, $\mathrm{R}$ the photodetector responsivity, $\mathrm{v}_{\mathrm{n}}$ the additive white Gaussian noise (AWGN) with variance $\sigma_{n}=\mathrm{N}_{0} / 2.0$, and $\mathrm{H}_{\mathrm{m}}$ the mth channel gain. The parameters of the two MISO topology channels are assumed to be equal for Simplicity, as are the parameters of the two arms SISO channel.

\section{Measuring performance}

The performance was measured based on Average (mean) BER (ABER) and can be computed using the results from [27] and expressed as: 
$A B E R=\int_{0}^{\infty} B E R_{\text {sim }}(H) \times f_{H}(H) d H$

where $B E R_{\text {sim }}(H)$ is the conditional simulated BER. The $f_{H}(H)$ is the channel gain $\mathrm{H}$ Probability Distributed Function (PDF). In weak turbulence, the log-normal distribution is applied. For the SISO channel, the PDF of the channel gain $\mathrm{H}$ is as mentioned in [25], while the PDF of the MISO and SISO channels are similar except dividing the variance $\sigma_{x}$ by number of transmitting elements [28]. The ABER is counted independently and thus whole variation can be calculated as:

$$
T A B E R=1-\prod_{i=1}^{N}\left(1-A B E R_{i}\right) \quad i=1,2, \ldots \ldots \ldots . . N
$$

i represents the ith drone, $\mathrm{N}$ represents the number of drones equal to the number of channels in the proposed system, and ABERi represents the average bit error rate for each channel..

\section{Results and discussion}

When setting the distance between the two drones in the same arm $60 \mathrm{~m}$, the distance between the GS and $D_{M} 1000 \mathrm{~m}$, and the altitude can vary from $1 \mathrm{~m}$ to $1000 \mathrm{~m}$, the performance of each subsystem against the system BER shown in Fig. 2, which demonstrate the operation of subsystems and whole system with $\mathrm{ABER} \approx 10^{-8}$.

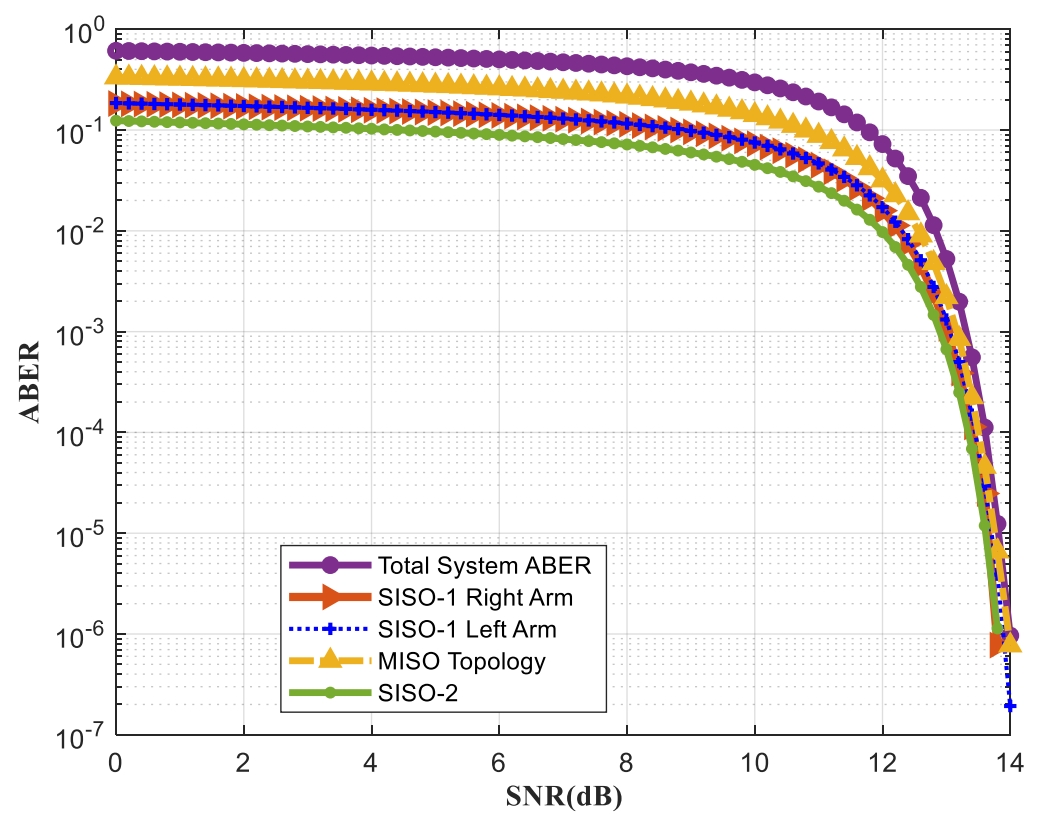

Fig. 2. Subsystems compared to whole system operation. 
To see the SNR differences between the subsystems and the overall system, setting the $\mathrm{ABER}=10^{-4}$ as a reference value, the difference in SNR for each part that is shown in Fig. 3 .

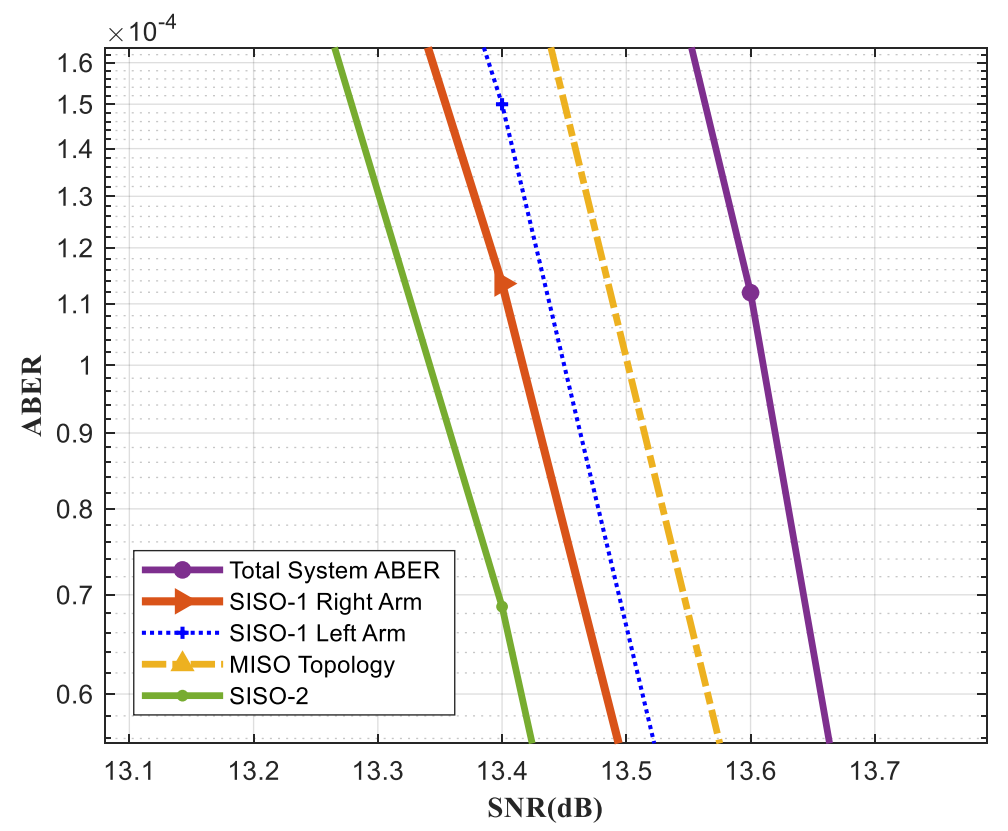

Fig. 3. The SNR differences between the subsystem and the overall system.

Fig 3 clarified that as the number of drones increased in the system, the ABER increased; therefore, the SNR values increased to get high performance. For example, if the system has only one drone, the SNR is $13.349 \mathrm{~dB}$, whereas when the system has five drones, the SNR is $13.62 \mathrm{~dB}$; this increases in SNR is to compensate for the increase in the bit errors of the five drones and have the same performance as for one drone. Table 2 summarized the SNR differences in numbers for each part of the flying system and the whole system is shown in Table 2.

Table 2. Differences in SNR for the whole system and the subsystems.

\begin{tabular}{ccc}
\hline System Part & Number of Drones & SNR $(\mathrm{dB})$ \\
\hline The whole system & 5 & 13.62 \\
MISO topology & 3 & 13.50 \\
SISO-1 left arm & 2 & 13.45 \\
SISO-1 right arm & 2 & 13.425 \\
SISO-2 & 1 & 13.349 \\
\hline
\end{tabular}

The subsequent test examined the system performance as the link distance $\mathrm{Z}$ increased more than $1000 \mathrm{~m}$. For example, link distances $Z=2000,6000$, and $8500 \mathrm{~m}$ as samples. In this case, the flying drones are considered a single system connected optically to the GS; therefore, we 
take the whole system's total average BER (TABER). The flying system becomes perpendicular to the GS when the altitude $h$ equals the link distance $Z$ value; therefore, this test can be generalized to the $Z$ and $h$ respectively distance and altitude $h$. Fig 4 showed that at SNR< $10 \mathrm{~dB}$, all the distances (or altitude $h$ ) showed the worst performance. After $S N R \geq 10 \mathrm{~dB}$, there was an enhancement in the performance until TABER $\approx 10^{-7}$ for distances $Z$ or $h=2000 \mathrm{~m}$ and $6000 \mathrm{~m}$. When $Z$ or $h$ increases, the system performance decreases, and vice versa. Table 3 summarized the SNR differences when setting TABER $=10^{-4}$.

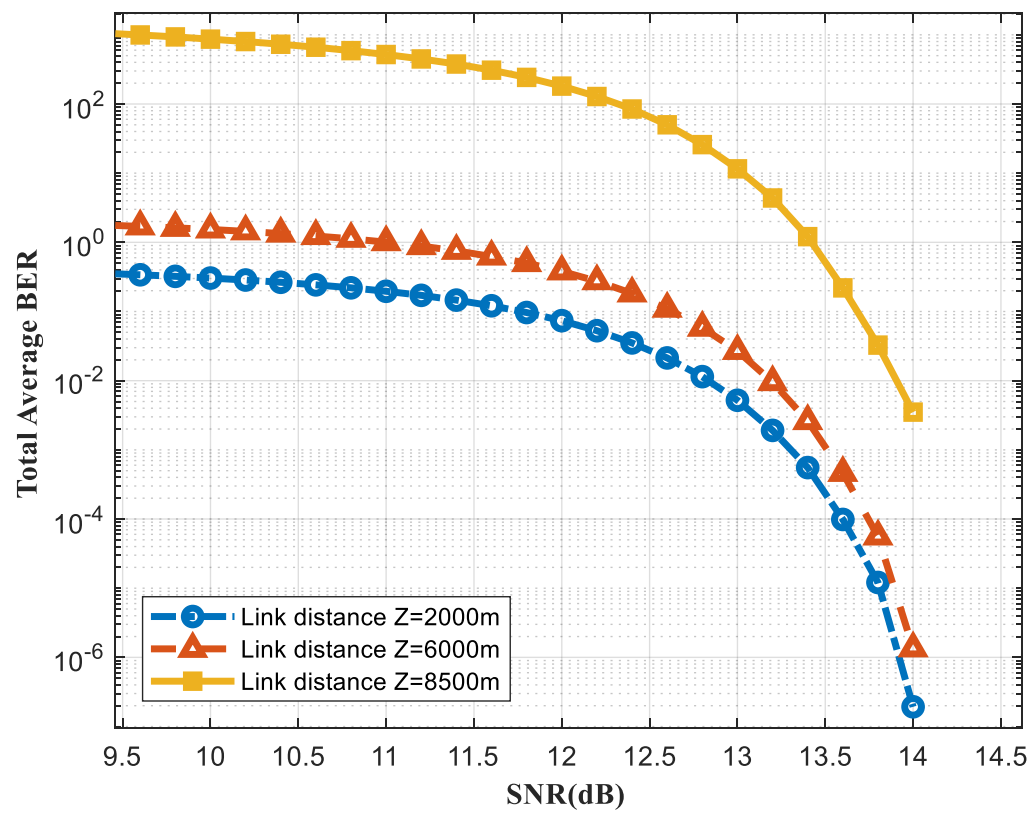

Fig. 4. System performance at various $Z$ - distances.

Table 3. Outcomes when the distance $Z$ or the altitude $h$ increases.

\begin{tabular}{|c|c|}
\hline $\begin{array}{c}\text { The link distance } \mathrm{Z} \text { or } \\
\text { the altitude } h(\mathrm{~m})\end{array}$ & SNR $(\mathrm{dB})$ \\
\hline 2000 & 13.601 \\
\hline 6000 & 13.75 \\
\hline 8500 & - \\
\hline
\end{tabular}

At $Z=8500 \mathrm{~m}$, the system showed the highest BER; therefore, $Z$ must be $<8500 \mathrm{~m}$; more specifically, $Z=7800 \mathrm{~m}$ got high performance for the values given parameter. The later distance can be applied between two drones and not just between the flying and GS. Also, the flying system can reach the highest altitude $h$ at $7800 \mathrm{~m}$, and the performance keeps high.

\section{Conclusions}


The ABER for each subsystem was determined, followed by the total ABER for the entire system. Each subsystem can be considered an independent system; then, the subsystems built the flying system. The results showed that as the number of drones increased, the SNR increased also. At SNR $<10 \mathrm{~dB}$, all of the results revealed that the ABER improves marginally. There are considerable increases in system performance after $S N R \geq 10 \mathrm{~dB}$. The maximum distance $Z$ and altitude $h$ could the flying system reach and got high performance was $7800 \mathrm{~m}$. Increases in the link distance $\mathrm{Z}$ are used to determine the maximum distance that the drones can travel for the characteristics listed in Table 1 . The beam becomes wider as the connecting distance $Z$ increases. A portion of the incident light is captured by the receiver. When the performance of the link degrades, the controller can change the link distance Z. For future study, critical factors affecting system performance, such as transmission power $\mathrm{P}_{\mathrm{T}}$ and receiver aperture diameter $\mathrm{D}_{\mathrm{R}}$, can be tuned to achieve ideal values.

\section{References}

[1] J. Hu and A. Lanzon, "An innovative tri-rotor drone and associated distributed aerial drone swarm control," Rob. Auton. Syst., vol. 103, pp. 162-174, 2018.

[2] C. Yan, L. Fu, J. Zhang, and J. Wang, "A Comprehensive Survey on UAV Communication Channel Modeling," IEEE Access, vol. 7, pp. 107769-107792, 2019.

[3] C. Koparan, A. B. Koc, C. V. Privette, C. B. Sawyer, and J. L. Sharp, "Evaluation of a UAVassisted autonomous water sampling," Water (Switzerland), vol. 10, no. 5, 2018.

[4] M. A. Khalighi and M. Uysal, "Survey on free space optical communication: A communication theory perspective," IEEE Commun. Surv. Tutorials, vol. 16, no. 4, pp. 2231-2258, 2014.

[5] S. Bloom, E. Korevaar, and J. Schuster, "Understanding the performance of free-space optics [Invited]," vol. 2, no. 6, pp. 178-200, 2003.

[6] H. Kaushal and G. Kaddoum, "Optical Communication in Space: Challenges and Mitigation Techniques," IEEE Commun. Surv. Tutorials, vol. 19, no. 1, pp. 57-96, 2017.

[7] I. S. Ansari, F. Yilmaz, and M. S. Alouini, "Impact of pointing errors on the performance of mixed RF/FSO dual-hop transmission systems," IEEE Wirel. Commun. Lett., vol. 2, no. 3, pp. 351-354, 2013.

[8] M. Sharma, D. Chadha, and V. Chandra, "Performance Analysis of Multihop Free Space Optical Communication System with Pointing Errors," J. Opt. Commun., vol. 36, no. 1, pp. 24-27, 2015.

[9] G. Immadi, M. Venkata Narayana, A. Sree Madhuri, and V. L. Tejaswani Sabbasani, "Simulation of free space optical communication under different weather conditions," Int. J. Pure Appl. Math., vol. 117, no. 18 Special Issue, pp. 143-148, 2017.

[10] W. Fawaz, C. Abou-rjeily, and C. Assi, "UAV-Aided Cooperation for FSO Communication Systems," IEEE Communications Magazine, no. January, pp. 70-75, 2018.

[11] E. Leitgeb, K. Zettl, S. S. Muhammad, N. Schmitt, and W. Rehm, "Investigation in free space optical communication links between unmanned aerial vehicles (UAVs)," Proc. 2007 9th Int. Conf. Transparent Opt. Networks, Ict. 2007, vol. 3, no. 1, pp. 152-155, 2007.

[12] J.-B. Wang, M. Sheng, X. Song, Y. Jiao, and M. Chen, "Comments on `BER Performance of FSO Links over Strong Atmospheric Turbulence Channels with Pointing Errors'," IEEE Commun. Lett., vol. 16, no. 1, pp. 22-23, Jan. 2012.

[13] S. Sheikh Muhammad et al., "Challenges in establishing free space optical communications between flying vehicles," in Proceedings of the 6th International Symposium Communication Systems, Networks and Digital Signal Processing, CSNDSP 08, 2008, pp. 82-86, 2008.

[14] A. Hatziefremidis, K. E. Zarganis, H. C. Leligou, and N. Pleros, "Bit error rate analysis along a slanted path link between UAVs and Ground Stations," 15th International Conference on Transparent Optical Networks (ICTON), pp. 1-4, 2013.

[15] W. J. Mazher, H. T. Ibrahim, O. N. Ucan, and O. Bayat, "Drone swarm with free-space optical communication to detect and make deep decisions about physical problems for area surveillance," 
Optical Engineering, vol. 57, no. 3, March 2018.

[16] M. T. Dabiri and S. M. S. Sadough, "Optimal Placement of UAV-Assisted Free-Space Optical Communication Systems With DF Relaying," IEEE Commun. Lett., vol. 24, no. 1, pp. 155-158, Jan. 2020.

[17] C. Chlestil et al., "Optical Wireless on Swarm UAVs for High Bit Rate Applications," IEEE CSNDSP, 2006, no. July 2015, pp. 634-638, 2015.

[18] S. S. Muhammad et al., "Challenges in establishing free space optical communications between flying vehicles," 6th International Symposium on Communication Systems, Networks and Digital Signal Processing, pp. 82-86, 2008.

[19] A. A. Khuwaja et al., "A Survey of Channel Modeling for UAV Communications," IEEE Commun. Surv. Tutorials, vol. 20, no. 4, pp. 2804-2821, 2018.

[20] C. R. Shah, "Performance and Comparative Analysis of SISO ,SIMO,MISO, MIMO," Int. J. Wirel. Commun. Simul., vol. 9, no. 1, pp. 1-14, 2017.

[21] N. a Mohammed, A. S. El-wakeel, and M. H. Aly, "Pointing Error in FSO Link under Different Weather Conditions," Int. J. Video Image Process. Netw. Secur., vol. 12, no. 1, pp. 6-9, 2012.

[22] M. T. Dabiri, S. Mohammad, S. Sadough, and M. A. Khalighi, "Channel Modeling and Parameter Optimization for Hovering UAV-Based Free Space Optical Links," IEEE J. Sel. Areas Commun., vol. 36, no. 9, pp. 2104-2113, 2018.

[23] T. Song, Q. Wang, M.-W. Wu, T. Ohtsuki, M. Gurusamy, and P.-Y. Kam, "Impact of Pointing Errors on the Error Performance of Intersatellite Laser Communications," J. Light. Technol., vol. 35, no. 14, pp. 3082-3091, Jul. 2017.

[24] M. T. Rahman, S. Iqbal, and M. M. Islam, "Modeling and performance analysis of free space optical communication system," International Conference on Informatics, Electronics and Vision, ICIEV 2012, no. April 2019, pp. 211-218.

[25] A. A. Farid and S. Hranilovic, "Outage capacity optimization for free-space optical links with pointing errors," J. Light. Technol., vol. 25, no. 7, pp. 1702-1710, 2007.

[26] K. Prabu, D. S. Kumar, and R. Malekian, "BER analysis of BPSK-SIM-based SISO and MIMO FSO systems in strong turbulence with pointing errors," Optik (Stuttg)., vol. 125, no. 21, pp. 6413-6417, Nov. 2014

[27] J. B. Wang, M. Sheng, X. Song, Y. Jiao, and M. Chen, "Comments on 'BER performance of FSO links over strong atmospheric turbulence channels with pointing errors,"' IEEE Commun. Lett., vol. 16, no. 1, pp. 22-23, 2012.

[28] M. Abaza et al., "MIMO Techniques for High Data rate Free Space Optical Communication System in Log-Normal Channe," The International Conference on Technological Advances in Electrical, Electronics and Computer Engineering, TAEECE 2013, pp. 1-5, 2013. 